\title{
Os Primórdios da Antropologia Brasileira \\ (1900-1979)
}

In memória de Denise Maldi.

\author{
Roque de Barros Laraia \\ Professor Emérito da UnB \\ Pesquisador Emérito do CNPq
}

Resumo: Toda história, como também os mitos, possuem muitas versões e o mesmo ocorre com a história da antropologia brasileira, principalmente no que se refere à origem formal da nossa disciplina. Pode ser que alguém acredite que nossa Antropologia começou nos austeros corredores do Museu Nacional, por onde entre vitrines repletas de ossos e de estranhos instrumentos, transitavam os primeiros antropólogos físicos. Outros podem transferir esse começo para a Semana Modernista de 22, onde pontificava a figura genial de Mário de Andrade, dublê de turista amador e pesquisador de campo. Mas eu, como minha colega, Mariza Corrêa, acredito que o nosso primeiro antropólogo foi o médico maranhense, radicado na Bahia, Raymundo Nina Rodrigues. Nesse sentido, é muito oportuno relembrar o papel dos médicos na história de nossa disciplina. Foram eles os fundadores ou incentivadores da Antropologia em diversas regiões do país. Assim, o que pretendo apresentar aos nossos é apenas uma versão da origem formal de nossa disciplina.

Palavras-chave: História; fundadores; antropologia; brasileira. 


\section{The Beginnings of the Brazilian Anthropology (1900-1979)}

Summary: Every story, as well as the myths, have many versions and so does the history of Brazilian anthropology, especially as regards the formal origin of our discipline. Can anyone believe that our anthropology began in the austere halls of the National Museum, where, between shop windows full of bones and strange instruments, transited the first physical anthropologists. Others may start to transfer this to the Modernist Week 22, where pontificated genial figure of Mario de Andrade, stunt of amateur tourist and fieldworker. But I, like my colleague, Mariza Corrêa, believe that our first anthropologist was a doctor from the State of Maranhão, based in Bahia, Raymundo Nina Rodrigues. Therefore, it is very timely to recall the role of doctors in the history of our discipline. They were the founders and supporters of Anthropology in various regions of the country. So, what I intend to present is our version of the formal source of our discipline.

Keywords: History; founders; anthropology; Brazilian.

\section{Los inicios de la antropología brasileña (1900 - 1979)}

Resumen: Toda historia, así como los mitos, tienen muchas versiones y lo mismo ocorre com la historia de la antropologia brasileña, especialmente em cuanto al origen formal de nuestra disciplina. Puede ser que alguien crea que nuestra Antropología comenzó em los pasillos austeros del Museo Nacional, donde entre as vitrinas llenos de huesos y extraños instrumentos, rabió los primeiros antropólogos físicos. Otros podrán transferir este principio a la Semana Modernista de 22, donde creen que ha pontificado la figura genial de Mário de Andrade, aficionado turístico y investigador de campo. Pero, al igual que mi colega, Mariza Corrêa, creo que nuestro primer antropólogo era el médico de Maranhão, nacido em Bahia, Raymundo Nina Rodrigues. En este sentido, es muy oportuno recordar el papel de los médicos en la história de nuestra disciplina. Eran los fundadores o exhortadores de Antropología em varias regiones del país. Entonces, lo que pretendo presentar a nuestro es sólo uma versión del origen formal de nuestra disciplina.

Palabras clave: historia; fundadores; Antropología; Brasileño. 
Toda história, como também os mitos, possuem muitas versões e o mesmo ocorre com a história da antropologia brasileira, principalmente no que se refere à origem formal da nossa disciplina. Assim o que pretendo apresentar aos nossos é apenas uma versão. Porém, antes de entrar propriamente neste texto, gostaria - como se fosse um prólogo ou até mesmo uma anedota - responder a questão: quando o termo antropologia foi utilizado primeiramente em relação ao nosso país? Vamos encontrar a resposta na segunda metade do século XIX, no livro Viagem do Rio de Janeiro a Morro Velho, Editora Itatiaia, Belo Horizonte, 1976, (edição inglesa, 1869) de autoria do destemido explorador britânico Richard Burton (1821-1869). A partir da página 322 ele apresenta as suas observações "sobre a antropologia descritiva de Minas Gerais". Nesse texto, Burton utiliza de conceitos de uma antropologia biológica da primeira metade do século XIX, que admitia a influência da fenotipia nas diferentes formas de comportamentos. Diz ele, então:

A tez do mineiro é de um pardo escuro morno, raramente corado na face, e muitas vezes perturbado pela secreção de bílis, ou pela obstrução dos condutos. É na realidade, a cor do português do Algarves, onde o mouro viveu durante tanto tempo.

A seguir, comparando os mineiros com os ingleses, ele afirma: "Entre nós (os ingleses) o temperamento nervoso é mais conhecido pelo cabelo sedoso e fino, aqui temos o mesmo acompanhado por um topete”.

Retomando o nosso tema, pode ser que alguém acredite que a nossa Antropologia começou nos austeros corredores do Museu Nacional, por onde entre vitrines repletas de ossos e de estranhos instrumentos, transitavam os primeiros antropólogos físicos, substituídos depois por Edgar Roquette-Pinto, Heloísa Alberto Torres e Raymundo Lopes. Outros podem transferir esse começo para a Semana Modernista de 22, onde pontificava a figura genial de Mário de Andrade, dublê de turista amador e pesquisador de campo. Mas eu, como minha colega, Mariza Corrêa, acredito que o nosso primeiro antropólogo foi o médico maranhense, radicado na Bahia, Raymundo Nina Rodrigues, que iniciou por estudar a procedência dos negros trazidos da África para a Bahia. A partir de uma postura inicial - que pode ser rotulada de racista - transformouse em um forte defensor dos ritos e dos costumes africanos. O seu livro Africanos no Brasil é, provavelmente, o primeiro texto antropológico produzido por um autor brasileiro.

Nesse sentido, é muito oportuno relembrar o papel dos médicos na história de nossa disciplina. Foram eles os fundadores ou incentivadores da Antropologia em diversas regiões do país. Assim foi com Roquette-Pinto e Arthur Ramos no Rio de Janeiro; Nina Rodrigues e Thales de Azevedo, na 
Bahia; Oswaldo Cabral, em Santa Catarina; José Loureiro Fernandes, no Paraná e René Ribeiro, em Pernambuco.

Inquirido a respeito deste fato, em entrevista concedida a Hélgio Trindade, o professor Luiz de Castro Faria respondeu:

Com a criação das Faculdades de Filosofia, Ciências e Letras todas elas eram obrigados a ter professores de antropologia, (...) no primeiro ano era antropologia biológica, então era um médico escolhido sempre, porque tinha a formação que se prestava a essa ampliação, em termos de raça, crânio...

A resposta em parte é verdadeira, mas os médicos antropólogos mencionados acima não podem ser definidos, apenas, como antropólogos físicos.

Recorro inicialmente a um texto de Florestan Fernandes, publicado na antiga revista Anhembi (números 72, 73, 74, 1956-1957) "Tendências teóricas da moderna investigação etnológica no Brasil”, no qual o referido autor discutia a existência ou não de um padrão intelectual de trabalho científico no Brasil. Para que esse desenvolvimento ocorresse, Fernandes postulava a necessidade da existência de centros de difusão do pensamento e do conhecimento científico. Para ele, o treinamento sistemático do pesquisador é a primeira condição, e a mais importante de todas, para um contínuo aperfeiçoamento de padrões intelectuais definidos de investigação científica. Acrescenta, então, que esse treinamento "repousa no ensino universitário, mas só se completa depois na experiência concreta de pesquisas".

Tudo isto que - decorridos hoje 58 anos da publicação do referido artigo nos parece simples, era uma tarefa extremamente difícil naquela época, porque no Brasil as universidades surgiram muito tardiamente. Essa lacuna teve que ser preenchida pelos Museus. Enquanto a nossa primeira Universidade somente foi criada em 1934, o Museu Nacional foi fundado em 1818; o Museu Paraense Emílio Goeldi, em 1866; o Museu Paranaense, em 1876; e o Museu Paulista, em 1895. Assim a pesquisa antropológica já dispunha de um espaço institucional desde o final do século XIX.

Esta afirmação pode ser considerada temerária, mas basta rever a história da nossa disciplina nas Universidades para verificar que na mais antiga delas, a Universidade de São Paulo, o ensino da Antropologia se desenvolveu mais lentamente do que se podia imaginar.

Maria Hermínia Tavares de Almeida (1989) afirma que "o início da institucionalização das ciências sociais no país data dos anos 30 e está associado às grandes transformações políticas e institucionais desencadeada pela revolução de 1930”. Em São Paulo, em 1933, um ano depois de o Estado ter sido derrotado na Revolução Constitucionalista, ocorreu a fundação da Escola Livre de Sociologia e Política, iniciativa de destacadas personalidades civis "como Roberto Simonsen, Jorge Street, Armando Sales de Oliveira Pacheco e Silva, Raul Piquet e André Dreifus" (Limongi, 1989). Somente no ano seguinte, 1934, foi criada a Universidade de São Paulo, um projeto da elite paulista, destacandose Julio Mesquita Filho, Paulo Duarte, Fernando de Azevedo, entre outros. 
Entretanto, foi no âmbito da Escola Livre de Sociologia e Política que, em 1939, as ciências sociais tomaram um grande impulso. Isto se deu com a chegada de Donald Pierson, recém-doutorado pela Universidade de Chicago. Em 1941, ele criou a Pós- Graduação em Ciências Sociais. Contava então com a colaboração de Emílio Willems e Herbert Baldus. Desde o início de 1942 até os meados de 1944, a Escola Livre de Sociologia e Política contou também com a participação de nada menos que A. R. Radcliffe-Brown. A presença do famoso antropólogo britânico, contemporâneo de Bronislaw Malinowski, atraiu um significativo número de estudantes, regulares ou ouvintes, entre eles Virginia Bicudo, Lucila Hermann, Oracy Nogueira, Egon Schaden e Virginia Watson (Melatti, 1978).

Abro um parêntese para comparar o significado da presença de RadcliffeBrown na Escola Livre de Sociologia e Política com a de Claude Levi-Strauss, na Universidade de São Paulo. Enquanto, Levi-Strauss era apenas um recémgraduado - que dava os primeiros passos de sua magnífica carreira acadêmica Radcliffe-Brown, trinta anos antes tinha realizado a sua pesquisa entre os Andamaneses, no Pacífico, e publicado a sua famosa monografia e mais de 60 artigos.

Voltando a Escola Livre de Sociologia e Política, e a título de curiosidade, utilizando uma informação de Donald Pierson (Corrêa, 2013) relacionamos as disciplinas do primeiro ano de pós-graduação: "Pesquisas Sociais na Comunidade Paulista" (Pierson); "Etnologia Brasileira” (Baldus); "Assimilação e Aculturação no Brasil Meridional" (Willems); além de três Seminários: "Métodos e Pesquisas Sociais" (Pierson e Rodolfei); "Índios do Brasil" (Baldus) e "Raça e Cultura", Pierson (cf. Corrêa, 2013). Entre os primeiros mestrandos da Escola Livre de Sociologia e Política podemos relacionar: Gioconda Mussolini, Lavínia Costa, Florestan Fernandes, David Maybury-Lewis e João Batista Borges Pereira.

Passo agora ao desenvolvimento da Antropologia na Universidade de São Paulo, que teve como ocupantes da Cátedra, Emílio Willems e a partir de 1949, Egon Schaden. Em 1945, ocorreram as defesas das duas primeiras teses de doutorado em Antropologia: Egon Schaden, "Ensaio Etno-Sociológico sobre a mitologia heróica de algumas tribos indígenas do Brasil", banca presidida por Fernando de Azevedo; e a tese de Lavínia Costa Raymond, "Algumas danças populares do Estado de São Paulo”, presidida por Roger Bastide.

Surpreendentemente, um espaço de tempo de 20 anos transcorreria até a aprovação da primeira dissertação de mestrado em Antropologia na Universidade de São Paulo: Eunice Ribeiro Durhan, "Mobilidade e assimilação: a história do migrante italiano em um município paulista”, 1964. Dois anos depois, a terceira tese de doutorado foi defendida também por Eunice Ribeiro Durhan.

A partir dessa data até o final de 1972 foram realizadas doze defesas de dissertação e sete de doutoramento. A explicação para este brusco aumento foi a promulgação em 20 de dezembro de 1962 da Lei 4024, denominada Lei de 
Diretrizes e Bases da Educação Nacional que modificou radicalmente a educação superior, extinguindo a Cátedra além de modificar o sistema seriado de cursos substituindo-o pelo de créditos. E em 3 de dezembro de 1965, o Parecer 977 do Conselho Federal de Educação - mais conhecido como Parecer Sucupira - regulamentou os cursos de Mestrado e Doutorado estabelecendo o sistema atual. Nessa ocasião, a Universidade de São Paulo estabeleceu o dia 31 de dezembro de 1972 como prazo final para as defesas dos inscritos no sistema anterior.

Iniciei o presente texto referindo-me a um artigo de Florestan Fernandes, meu orientador de tese de doutorado. Dou prosseguimento ao mesmo citando um artigo de Roberto Cardoso de Oliveira, a quem devo a minha formação básica em Antropologia e quem também foi orientando de Florestan, em seu artigo "O que é isso que chamamos de antropologia brasileira?" (Anuário Antropológico, 1985) ele chama a atenção para o fato que, no Brasil, a nossa disciplina era primordialmente denominada de Etnologia citando o título do artigo de Florestan Fernandes já mencionado. Esta sua afirmação nos leva a algumas considerações, sendo uma delas a forte presença de docentes franceses na USP. Como sabem na Europa continental o termo "antropologia” estava reservado à Antropologia Biológica. Por outro lado, seguindo a argumentação de Cardoso de Oliveira a utilização do termo Etnologia era, entre nós, inicialmente vinculada ao predomínio dos estudos de nossas sociedades indígenas. Tal fato teria levado, posteriormente, à uma divisão temática, como Etnologia das Sociedades Indígenas e Antropologia da Sociedade Nacional.

Creio que esta rotulação é hoje uma questão totalmente superada. Faz muito tempo que a nossa comunidade científica optou pelo termo Antropologia, que designa tanto a nossa Associação Científica, como nossos cursos de graduação e pós-graduação e também os nossos títulos acadêmicos.

Em seu texto, acima citado, Roberto Cardoso de Oliveira nos forneceu uma periodização da história da Antropologia no Brasil. Denominou como heroico o primeiro período, ou seja, o tempo que "corresponde às décadas de 20 e 30 quando a profissão de antropólogo e o próprio campo da antropologia não estavam institucionalizados entre nós e, portanto, o trabalho de pesquisa tinha o sabor de uma atividade verdadeiramente heroica”. É o tempo de RoquettePinto, Raymundo Lopes e Heloísa Alberto Torres, no Museu Nacional; Arthur Ramos, no Rio de Janeiro; Gilberto Freyre, Carlos Estevão de Oliveira, no nordeste; de Herbert Baldus e Charles Wagley entre os Tapirapé, e, sobretudo, de Curt Nimuendajú, o hoje lendário fundador da etnologia brasileira. Cardoso de Oliveira referindo-se ao clima de heroísmo que se atribuia à pesquisa de campo cita o romance de Basto Ávila, "No Pacoval do Carimbé”, publicado em 1932, e que se refere à pesquisa de Heloisa Alberto Torres (1895-1977), realizada na Ilha de Marajó em 1929. Segundo ele, "mulher-antropóloga era então por demais inesperado"!

Cardoso de Oliveira denominou de carismático o período seguinte, que começa no final dos anos 40, “com a obra etnológica de Florestan Fernandes e 
as pesquisas de Darcy Ribeiro e Eduardo Galvão”. São eles as figuras centrais que reuniram em torno de si e de seus projetos jovens estudantes de antropologia. É importante salientar que Ribeiro preocupou-se, no final da década de 50, com a formação de novos quadros para a etnologia com a realização de dois cursos de indigenismo: o primeiro realizado no âmbito do Museu do Índio (criado por ele em 1953) e o segundo no Centro Brasileiro de Pesquisas Educacionais, contando no segundo caso com a cooperação de Roberto Cardoso de Oliveira. Poderíamos dizer hoje que foram cursos de especialização não fosse o fato que no primeiro deles não foi exigido dos alunos um diploma de nível superior.

É nesse período, que abrange o final dos anos 40 e toda a década seguinte, que vão ocorrer quatro fatos muito significativos na história de nossa disciplina.

Em 1953, no Museu Nacional, a realização da $1^{\text {a }}$ Reunião Brasileira de Antropologia, organizada por Heloísa Alberto Torres, tendo como presidente de honra Edgar Roquette-Pinto, e Luis de Castro Faria, como secretário. Participaram da Comissão Organizadora: Heloísa Alberto Torres, Eduardo Galvão, Luiz de Castro Faria, Pedro Lima e Tarcisio Messias. Uma bem conhecida foto mostra o público da $1^{\mathrm{a}}$ ABA: Loureiro Fernandes, Herbert Baldus, Mario Wagner Vieira da Cunha, Thales de Azevedo, René Ribeiro, Ego Schaden, Darcy Ribeiro, Heloísa Alberto Torres, Oracy Nogueira, Manuel Diegues Jr., Marina Vasconcelos, Edson Carneiro, Ruth Landes, Maria Julia Pouchert, Bastos Ávila e Luiz de Castro Faria, e alguns poucos "desconhecidos" totalizando trinta pessoas.

Nesse mesmo ano, Darcy Ribeiro, então coordenador da Sessão de Estudos do Serviço de Proteção aos Índios, criou o Museu do Índio, em um prédio pertencente ao Ministério da Agricultura, ao lado do Maracanã.

Em 1954, em São Paulo, no Hotel Esplanada - situado então atrás do Teatro Municipal, realizou-se o XXXI Congresso Internacional de Americanistas, pela primeira e única vez realizado no Brasil.

Finalmente em 1955, durante a $2^{\text {a }}$ Reunião Brasileira de Antropologia, realizada em Salvador, foi criada a nossa Associação Brasileira de Antropologia. Duas tentativas anteriores fracassaram: a Sociedade de Etnografia e Folclore e a Sociedade Brasileira de Etnologia.

Nos anos 50, continua o predomínio da influência americana no Brasil. É o período dos chamados "estudos de comunidades", todos eles inspirados na obra clássica de Robert e Helen Lynd, Middletown. A in American Study (Harcourt Brace and Company, New York, 1929). Foram publicadas mais de uma dúzia de monografias entre as quais destacamos as seguintes: Emilio Willems, Cunha, Tradição e Transição em uma Cultura Rural do Brasil, São Paulo, 1948; Donald Pierson, Cruz das Almas. A Brazilia Village, Washington, 1951; Emilio Willems e Gioconda Mussolini, Buzios Island. A Caiçara Community in Southern Brazil, Nova York, 1952; Charles Wagley, Amazon Town. A Study of Man in the Tropics, Nova York, 1953; Fernando Altenfelder Silva, Análise Comparativa de Alguns Aspectos da Estrutura Social de duas Comunidades do 
Vale do São Francisco, Curitiba, 1955, Marvin Harris; Town and Country in Brazil, Nova York, 1956; etc.

Muitas foram as críticas feitas aos estudos de comunidades, como por exemplo a de Otávio Ianni, "Estudo de Comunidade e Conhecimento Científico" na Revista de Antropologia, vol. 9, nº 1 e 2 , 1961, mas é inegável a importância dos mesmos para o conhecimento da realidade brasileira. Em 1962, a antropóloga Maria Laís Mousinho Guidi publicou na revista Educação e Ciências Sociais v.10, n.19, o seu trabalho "Elementos de Análise dos Estudos de comunidades realizados no Brasil e publicados de 1948 a 1960" que se tornou uma leitura obrigatória para quem quiser conhecer essa fase de nossa disciplina.

Desde os anos 30, diversos estudos antropológicos têm o negro como objeto de estudo. É o caso de Gilberto Freyre, em Casa Grande e Senzala, 1933, uma importante e polêmica interpretação do Brasil, que continua sendo objeto de discussões até os nossos dias. É dessa década também o trabalho de Donald Pierson, que resultou no livro Branco e Pretos na Bahia (1945). Mas os estudos antropológicos sobre os negros vão se intensificar na década de 50.

Voltamos, então, a Roberto Cardoso de Oliveira que cita um terceiro período que denominou de burocrático que chega até o presente, tendo o seu início na segunda metade dos anos 60, e coincide com a criação dos cursos de mestrados no país, segundo as normas do parecer Sucupira. Neste último período - que eu prefiro chamar de moderno - diminuiu sensivelmente a possibilidade da existência de antropólogos autodidatas, como por exemplo Curt Nimuendajú. Muito contribuiu para isto a valorização dos títulos de pósgraduação. Até o final dos anos 60, o rito de iniciação do antropólogo era a sua primeira pesquisa de campo. Atualmente é a obtenção do título de doutor!

Não há dúvida que os anos 60 constituíram um marco importante do crescimento do papel das Universidades na formação de novos antropólogos, mas caberia ainda a um Museu uma importante iniciativa. Em 1960, Cardoso de Oliveira - juntamente com Luiz de Castro Faria - criou o "Curso de Teoria e Pesquisa em Antropologia Social - realizado no Museu Nacional, com a cooperação do Instituto de Ciências Sociais, da Universidade do Brasil. Tratavase da primeira edição de um curso de especialização, com um ano de duração, que exigia de seus alunos uma dedicação integral. O importante é que, no decorrer do mesmo, os alunos participavam de um trabalho de campo em uma área indígena, acompanhado de um pesquisador sênior. $\mathrm{O}$ mesmo foi responsável pela iniciação antropológica de onze pesquisadores: Alcida Ramos, Edson Soares Diniz, Roberto Augusto da Matta, Roque de Barros Laraia, Julio Cezar Melatti, Maria Andréa Loyola, Marcos Magalhães Rubinger, Maria Cecília Vieira Helm, Maria Stella Amorim e Silvio Coelho dos Santos, além de dois outros que não seguiram a carreira acadêmica.

Quase que simultaneamente com a criação do curso de especialização, Cardoso de Oliveira inicia o projeto "Estudos das Áreas de Fricção Interétnica no Brasil" que contou com a participação de alguns dos seus alunos do Curso de Especialização: Roberto Da Matta, Roque de Barros Laraia e Julio Cezar Melatti. 
Deste projeto resultaram os seguintes livros: O Índio e o Mundo dos Brancos, de Roberto Cardoso de Oliveira; Índios e Castanheiros, de Roque de Barros Laraia e Roberto DaMatta; Índios e Criadores, de Julio Cezar Melatti.

Em 1962, a Divisão de Antropologia do Museu Nacional firmou um convênio com a Universidade de Harvard para a execução do Harvard Central Brazil Reseach Project, coordenado por David Maybury-Lewis e Roberto Cardoso de Oliveira, que contou com a participação de vários jovens antropólogos americanos - Terence Turner, Joan Bamberger, Jean Carter Lave, Dolores Newton, J. Christofer Crocker, e Cecil Cook - além de Julio Cezar Melatti e Roberto DaMatta.

O golpe militar de 1964 atingiu pesadamente as Ciências Sociais brasileiras. O seu maior impacto foi, no entanto, no contexto das Universidades. A Antropologia foi menos atingida que a Sociologia, a Ciência Política e a História. A Cadeira de Sociologia da Universidade de São Paulo foi praticamente dizimada com as aposentadorias compulsórias de Florestan Fernandes, Fernando Henrique Cardoso e Otávio Ianni. A Reunião da Associação Brasileira de Antropologia que deveria ocorrer, em 1965, em Brasília, foi cancelada. Apesar disto foi no segundo lustro dos anos 60, em plena ditadura, que a Antropologia iniciou o seu grande crescimento, fato que levou Mariza Corrêa a perguntar: "como em um chão político tão inóspito pode florescer a planta da antropologia?".

Em julho de 1968 - seis meses antes do AI-5 - o Museu Nacional deu início ao primeiro Programa de Mestrado em Antropologia Social, em conformidade com as normas estabelecidas pela Portaria Sucupira. Esse fato somente foi possível graças ao empenho de Roberto Cardoso de Oliveira e à forte cooperação de David Maybury-Lewis, da Universidade de Harvard, que conseguiu o apoio da Fundação Ford. Foram selecionados 12 alunos, a metade deles provenientes da Pontifícia Universidade Católica do Rio de Janeiro. Eram eles: Alice Rangel, Claudia Menezes, Eurípedes Cunha Dias, Lucia Matoso, Lygia Sigaud, Madalena Diégues, Maria Andréa Loyola, Maria Rosilene Alvim, Neide Esterci, Otávio Velho, Paulo Marcos Amorim e Wagner Rocha.

Alguns dos alunos acima participaram de um projeto, também no âmbito do Convênio com a Universidade de Harvard visando o estudo comparativo das populações rurais das regiões Nordeste e Centro-Oeste. David Maybury-Lewis coordenou a pesquisa no Nordeste e Francisca Isabel Vieira Keller, no Brasil Central, mais precisamente no eixo da Belém-Brasília.

A década de 70 constituiu, sem dúvida, o momento mais importante na consolidação da disciplina no Brasil. Em 1972, foram criados os programas de pós-graduação em Antropologia Social da Universidade de Brasília e o da Universidade Estadual de Campinas. Nos anos seguintes da década, novos programas de pós-graduação surgem na Universidade Federal do Rio Grande do Sul, Universidade Federal de Pernambuco, e na Universidade Federal do Rio Grande do Norte. Ao lado das instituições acima citadas, o período final da década foi também marcado por centros de pesquisas antropológicas, ao lado do 
ensino lato sensu, como o Museu do Índio, no Rio de Janeiro; o Museu Paulista; o Instituto Joaquim Nabuco de Pesquisas Sociais, em Recife; além da Universidade Federal do Paraná. E, finalmente, o desenvolvimento ocorria também nos Programas de Pós-Graduação em Ciências Sociais, nos quais a Antropologia aparecia como uma área de concentração. Faziam parte desta categoria: o Instituto de Filosofia e Ciências Humanas da Universidade Federal da Bahia; o Departamento de Ciências Sociais da Universidade Federal de Santa Catarina; o Departamento de Ciências Sociais da Universidade Federal de Minas Gerais; a Pontifícia Universidade Católica de São Paulo; e o Instituto de Filosofia e Ciências Humanas da Universidade Federal do Rio de Janeiro.

Um levantamento realizado pelo $\mathrm{CNPq}$, no início da década seguinte, indicava que o corpo docente antropológico, nas instituições acima mencionadas, era de 164 professores, sendo 61 portadores do grau de doutor; 65 mestres, 14 livres-docentes, e 24 outros (?).

No que se refere às publicações especializadas, a década 70 marca o fim do monopólio da Revista de Antropologia e da Revista do Museu Paulista, bem como dos boletins de diferentes Museus. Em Brasília, fundado por Roberto Cardoso de Oliveira, surge o Anuário Antropológico, em 1976. A partir de então cada Programa foi criando a sua revista. É verdade que, antes disto, artigos de Antropologia podiam ser publicados em revistas como Dados, Revista de Ciências Sociais, Religião e Sociedade, etc.

Como vimos, até o início dos anos 70, a etnologia indígena e os estudos relacionados com o negro, constituíam os temas dominantes de nossa disciplina. Graças ao apoio de diferentes agências de financiamento de pesquisa - Finep, Fapesp, Fundação Ford e, principalmente, o Conselho Nacional de Pesquisas - surgem novas linhas de pesquisas: Antropologia da Sociedade Rural, Antropologia Urbana, Antropologia da Saúde, Identidade Étnica, Frentes de Expansão e Rituais e Simbolismo.

Além de tudo isto, a década de 70 foi palco de um ato político muito importante para a nossa história. Como todos sabem, a $6^{\text {a }}$ Reunião Brasileira de Antropologia, reunida em São Paulo, em 1963, elegeu como novo Presidente, Eduardo Galvão, então na Universidade de Brasília. Com o golpe de 1964, a demissão de Eduardo Galvão, da UnB, na crise de 1965, a $7^{\text {a }}$ RBA foi adiada. Ela só voltaria a ocorrer, em 1966, em Belém, durante as comemorações do Centenário do Museu Paraense Emílio Goeldi. Nessa reunião foi eleito o novo Presidente da ABA, o professor Manuel Diegues. Em 1971, graças aos esforços dos professores Egon Schaden e João Batista Borges Pereira, foi realizada em São Paulo, durante o Encontro Internacional de Estudos Brasileiros, a $8^{\mathrm{a}}$ RBA. Devido ao caráter semi-clandestino da reunião não foi possível eleger um novo presidente, que continuou sendo o Professor Diegues.

Finalmente, em 1974, graças ao empenho de Silvio Coelho dos Santos e Manuel Diegues Jr. foi possível a realização, em Florianópolis, da $9^{\text {a }}$ RBA. Como em todas as reuniões anteriores era esperado um número pequeno de participantes. Estes ultrapassaram a cifra dos 400! Em um tempo de passagens 
aéreas caras, numerosos ônibus chegaram a Florianópolis, procedentes do Nordeste e até mesmo de Belém do Pará. Os novos programas de pesquisa e pós-graduação marcaram assim a sua presença. Desde, então, as Reuniões Brasileiras de Antropologia vem se reunindo a cada dois anos. Na última, em 2012, em São Paulo, o número de participantes chegou a 4.00o!

Nos anos 70, o Brasil vivia o pior período da ditadura militar, mas apesar disto a antropologia cresceu significativamente nesse período. Como entender este paradoxo? Um importante fato que contribuiu para esse desenvolvimento foi, sem dúvida, o apoio da Fundação Ford que apoiou fortemente a criação do PPGAS do Museu Nacional, do PPGAS de Brasília, o Programa de PósGraduação em Ciências Sociais da Universidade Federal de Minas Gerais, além da criação do CEBRAP, em São Paulo, que abrigou vários dos aposentados compulsoriamente da USP. Em julho de 1977, a Fundação Ford participou ativamente da criação da ANPOCS que com suas reuniões anuais criou um importante espaço para os pesquisadores de Antropologia, além de seu papel importante de cooperação interdisciplinar.

Nos anos 70, a ABA não ficou só na luta em defesa dos direitos de nossas populações indígenas. Além da ANPOCS contou com a forte cooperação da SBPC em três memoráveis reuniões anuais (Brasília, Recife e São Paulo) com a participação de milhares de pessoas, tornando-se o principal espaço de protesto contra a ditadura. Em todas essas reuniões a questão indígena esteve em evidência.

De fato, desde o início da década, um forte grupo de antropólogos, predominantemente jovens, aproveitou todo o espaço possível para denunciar toda a violência cometida contra os índios. Assim marcaram as suas presenças no VI Congresso Interamericano de Indigenismo, realizado em Brasília, em 1972, bem como nas reuniões da ABA e da SBPC, como vimos.

Mas, o desafio maior estava por vir: em 1978, o Ministério do Interior apresentou um projeto de lei de Emancipação das Comunidades Indígenas. A ABA divulgou um documento de protesto contra o projeto mostrando que o Estado pretendia lavar as mãos de suas responsabilidades perante os índios. $\mathrm{E}$ utilizando de um discurso aparentemente generoso escondia o fato que a pretendida emancipação de fato entregaria os índios a "forças infinitamente mais poderosas que lhes arrebatarão em curto prazo as suas terras e os transformarão em mão de obra barata". Convencido dos argumentos apresentados, o general Ismarth de Araujo, Presidente da FUNAI, convidou os antropólogos Carmem Junqueira (PUC/SP), Cecilia Maria Vieira Helm (UFPR), Egon Schaden (USP), Lux Vidal (USP), Maria Manuela Carneiro da Cunha (USP), Pedro Agostinho da Silva (UFBA), Roberto Cardoso de Oliveira (UnB), Roberto da Matta (MN/UFRJ) e Silvio Coelho dos Santos (UFSC) para comparecerem a uma Reunião do Conselho Indigenista da FUNAI, nos dias 12 e 13 de setembro (estavam presentes, ainda, Roque de Barros Laraia (UnB) e Charlote Emerich (MN/UFRJ), representantes do Ministério da Educação no 
referido Conselho). Após a leitura do documento da ABA, ocorreu a votação pelos membros do Conselho e o projeto foi definitivamente arquivado.

Enfim, em um "chão político tão inóspito" como foram os anos 70. A antropologia brasileira se consolidou apesar das fortes dificuldades que teve que enfrentar. E os nossos colegas do exterior sabiam disso, como expressou Patricia Lyon, em seu livro Native South Americans (Little, Brown and Co. Boston, 1974): "Embora os problemas dos índios brasileiros tenham se tornado do conhecimento mundial apenas nos anos recentes, os antropólogos brasileiros há muito tempo têm conhecimento dos mesmos e tem tentado chamar a atenção para o problema e fazer alguma coisa para solucioná-lo". 


\section{Referências Bibliográficas}

BURTON, Richard. Viagem do Rio de Janeiro ao Morro Velho. Belo Horizonte, Editora Itatiaia, 1976.

CARDOSO DE OLIVEIRA, Roberto. O que é isso que chamamos de Antropologia Brasileira?. Anuário Antropológico /1985. Rio de Janeiro, Tempo Brasileiro, 1986.

CORREAA, Mariza. Traficantes do Simbólico \& Outros Ensaios sobre a História da Antropologia. Campinas, Editora Unicamp, 2013.

FERNANDES, Florestan. Tendências Teóricas da Moderna Investigação Etnológica no Brasil. Etnologia e Sociologia no Brasil. São Paulo, Editora Anhambi, 1958.

GUIDI, Maria Lais Mousinho. Elementos de Análise de Estudos de Comunidades Realizados no Brasil e Publicados de 1948 a 1960. Revista Educação e Ciências Sociais. Rio de Janeiro, vol. 10, n. 19, 1962.

IANNI, Otávio. Estudos de Comunidades e Conhecimento Científico. Revista de Antropologia, São Paulo, vol. 9, n. 1 e 2, 1961.

LIMONGI, Fernando. A Escola Livre de Sociologia e Política. In: MICELI, Sergio (org.). História das Ciências Sociais no Brasil. Rio de Janeiro: IDESP/FINEP, 1989.

LYON, Patricia (ed.). Native South Americans - Ethnology of the Least Known Continent. Boston/Toronto: Little, Brown and Company, 1974.

MELATTI, Julio Cezar. Introdução. In: MELATTI, J.C. (org.). Radcliffe-Brown: Antropologia. São Paulo: Ática, 1978.

NINA RODRIGUES, Raymundo. Africanos no Brasil. Brasília: Editora UnB, 2004 .

TAVARES DE OLIVEIRA, Maria Hermínia. Dilemas de Institucionalização das Ciências Sociais no Rio de Janeiro. In: MICELI, Sergio (org.). História das Ciências Sociais no Brasil. Rio de Janeiro: IDESP/FINEP, 1989.

TRINDADE, Helgio. Ciências Sociais no Brasil. Diálogos com Mestres e Discípulos. Brasília: ANPOCS/Interline Editora, 2012. 